\title{
面向复杂动态场景的多无人机协同摄影
}

\author{
崔云鹏，谢科，周漾，黄惠 ${ }^{*}$ \\ (深圳大学可视计算研究中心 深圳 518060) \\ (hhzhiyan@gmail.com)
}

\begin{abstract}
摘 要: 当前, 对复杂动态场景的实时拍摄仍然以人为操控的方式为主. 为了使用无人机对这种场景自动地进行完 整拍摄，提出将其分解为场景分割、拍摄构图和无人机调度 3 个子问题的方法. 首先采用带有阈值的 $K$-means 聚类算 法完整、有效地对场景进行合理分割，以获得一系列小场景，每个小场景对应一架无人机; 然后根据小场景之间的位 置关系计算获得无人机最佳的拍摄位置，保证在完整拍摄每个小场景的同时，无人机群拍摄的画面有一定的关联性 和整体性; 最后将无人机飞行调度问题转化为任务指派问题, 使用匈牙利算法将拍摄位置指派给每架无人机完成相 应的拍摄任务, 使无人机群的飞行距离总和最短, 完成对复杂动态场景的实时持续拍摄. 实验结果表明, 该方法可以 有效地使用仿真程序控制多架无人机，对复杂动态场景进行多角度且包含全部对象的实时持续自动拍摄. 通过观察 无人机的飞行轨迹和拍摄结果中目标物体的位置, 可以发现, 该方法相比场景均分等直接方式, 拍摄效果有明显的 提升.
\end{abstract}

关键词: 无人机；航空摄影；多视角；协同拍摄；虚拟仿真

中图法分类号: TP391.41 DOI: 10.3724/SP.J.1089.2021.18614

\section{Multi-UAVs Collaborative Cinematography for Complex Dynamic Scenes}

Cui Yunpeng, Xie Ke, Zhou Yang, and Huang Hui*

(Visual Computing Research Center, Shenzhen University, Shenzhen 518060)

\begin{abstract}
For real-time shooting of complex dynamic scenes, people still rely on controlling manually now. For automatically and completely shooting this kind of scene, a feasible solution is proposed, which divides the problem into $3 \mathrm{sub}$ problems: scene segmentation, photography composition, and UAV (unmanned aerial vehicle) scheduling. First, on scene segmentation, $K$-means clustering is utilized with threshold to get a series of small segmented scenes. Each small scene is corresponded by a single UAV. Then, according to the location of these small scenes, the best shooting positions of UAVs are calculated, which ensure that each small scene is shot completely and meanwhile the pictures shot by different UAVs are relevant and holistic. Finally, in order to minimize the total flight distance and flight cost of the UAV group, the UAV flight scheduling problem is turned into a task assignment problem, where Hungarian algorithm is applied to determine the final shooting positions for each UAV to complete its corresponding shooting task. By this way, a complex dynamic scene can be continuously shot in real-time. Experiments in simulation program shows that the algorithm can provide effective control on multiple UAVs to continuously and automatically shoot a complex dynamic scene, including all the objects inside the scene from multi-view in real-time. By observ-

收稿日期：2020-08-29; 修回日期：2020-11-16. 基金项目：国家自然科学基金(U2001206，61761146002); 广东省自然科学基金 (2020A0505100064，2015A030312015); 深圳市基础研究基金(JCYJ20180305125709986); 南山领航团队支持计划(20170003). 崔云鹏 (1994-), 男, 硕士, 主要研究方向为计算机图形学; 谢科(1982-), 男, 博士, 副研究员, CCF 会员, 主要研究方向为计算机图形学; 周漾(1986-), 男, 博士, 助理教授, CCF 会员, 主要研究方向为计算机图形学; 黄惠(1977一), 女, 博士, 教授, 博士生导师, CCF 杰 出会员, 论文通讯作者, 主要研究方向为计算机图形学.
\end{abstract}


ing trajectories of UAVs and objects' positions in shooting results, it is sure that proposed method produces results with much higher quality comparing with the naive method of average view division.

Key words: UAV (unmanned aerial vehicle); aerial photography; multi-view; collaborative cinematography; virtual simulation

随着无人机技术的深人发展，对运动目标跟 踪航拍已经成为无人机应用的研究热点之一. 在 复杂环境中, 采用无人机对地面动态目标进行实 时拍摄，在军事、交通、灾害和娱乐等多个领域发 挥重要作用. 但使用无人机拍摄动态场景比一般 的摄影系统更加复杂. 对于一个复杂的动态场景, 如对于一场多人的球类比赛或某个指定的活动广 场、公园等场地, 人们总是希望能完整、清晰地观 看整个场景的各个细节，尤其是其中的运动对象， 而人工控制相机拍摄这种大型场景是一项非常考 验摄影师技术的工作. 手动操纵无人机进行拍摄 一般至少需要 2 个专业人员进行协作, 他们分别控 制无人机和相机，拍摄成本高，同时无人机手动飞 行为安全起见和保证遥控器与无人机之间无线通 信的畅通, 无人机需要保持在目视范围内, 这限制 了无人机可以拍摄的范围. 因此, 采用无人机在空 中进行自动航拍是自然而然的想法. 理想的自动 航拍方法应该是基于用户的拍摄意图，生成平滑 的相机轨迹, 然后在环境和其他约束下进行拍摄. 这样的拍摄方法有很多富有挑战性的问题需要解 决, 包括拍摄任务的定义、将拍摄任务转化为飞行 任务、对环境的感知能力、无人机的任务分配及自 动应急能力等 ${ }^{[1-2]}$. 然而对于目标很多、范围较大 的场地, 很可能出现一架无人机的拍摄范围无法 完整覆盖整个场景, 或者只能从较远、较高的位置 对场景进行俯瞰式观察, 而难以获得关注对象的 运动细节等问题，此时就需要使用多个无人机对 这个场景进行拍摄. 场景中的物体有主有次, 因此 如果在拍摄过程中采取对场景进行均分, 然后让 每架无人机使用相同视角进行拍摄的方法, 将难 以得到令人满意的拍摄效果. 如果可以设计某种 算法能完整、有效地对场景进行合理分割, 然后对 多无人机系统分配拍摄任务并进行有效控制, 达 到实时跟踪拍摄场景中的主要对象的效果, 就可 以解决这种场景的拍摄问题.

\section{1 无人机航拍相关工作}

航拍又称为空中摄影或航空摄影 ${ }^{[3]}$. 无人机航
拍是以无人机为载体, 以机载遥感设备(如相机、 扫描仪、磁测仪等)获取信息, 然后用计算机对图 像信息进行处理，并按照一定精度要求制作成图 像. 无人机航拍由于其飞行器小巧灵活、机动高 效、成本低廉和便于智能化自动控制而在航拍领域 中占据日益重要的地位.

对一个场景使用无人机进行航拍，已经是一 项成熟且应用广泛的工作, 大量优秀的视频和影 片使用无人机航拍制作，如《鸟瞰世界》《航拍中 国》《大国重器》等. 然而, 以上纪录片中, 航拍的 对象以大尺度但较为固定的场景为主, 如建筑物 和风景名胜这种景观类场景. 场景中的物体不运 动或运动物体并不是拍摄的重点, 采用固定的、提 前生成的航拍路线进行航拍, 且由于拍摄影片的 性质, 可以进行反复拍摄, 对实时性要求偏低. 对 于大尺度且固定的场景进行传统航拍的作业流程, 如 2015 年毕凯等 ${ }^{[4]}$ 在轻小型无人机航拍的综述中 所述, 分为任务规划、航线设计、航摄飞行、质量 检查、补飞或重飞、现场预处理、像控测量、数据 处理和结果汇总等步骤. 如何生成航拍路线是这 个作业流程的核心问题. 该过程通常为在 3D 模拟 器中创建轨迹原型, 通过创建手动定位的关键帧 集合设计虚拟轨迹. Salomon 等 ${ }^{[5]}$ 和 Nieuwenhuisen 等 ${ }^{[6]}$ 很早就利用先验的 $3 \mathrm{D}$ 环境知识, 通过遍历 路线图计算出平滑的路径轨迹. Joubert 等 ${ }^{[7]}$ 通过要 求用户指定关键帧的时间，在关键帧之间创建一 个特定的连续轨迹, 用户可以不断地修改关键帧 时间, 以获得更好的航拍路径轨迹. Gebhardt 等 ${ }^{[8]}$ 提出了一种可以在虚拟环境中绘制和编辑摄像机 路径并进行优化以确保飞行轨迹可行性的设计工 具. Roberts 等 ${ }^{[9]}$ 杜杆式地优化了时间规划, 提高了 规划轨迹的可行性. Fleureau 等 ${ }^{[10]}$ 使用 Toric 空间 表示法表达摄影属性(距目标距离、目标角度、目 标的屏幕位置), 并自动计算移动目标之间的视点 转换. 2018 年, Xie 等 ${ }^{[11]}$ 就利用预先制作拍摄区域 的粗略 $2.5 \mathrm{D}$ 模型, 生成平滑且富有美感的无人机 轨迹, 利用该轨迹拍摄能连续捕捉大规模场景中地 标的视频. 然而, 这种拍摄方式并不适用于对复杂 动态且不可预测的场景进行实时拍摄. 
对于动态场景采用无人机进行自动拍摄，是 一项非常具有挑战性也比较新颖的工作. 跟踪实 时目标需要不断地对目标和环境同时更新预测, 生成一条可行轨迹, 然后据此执行飞行任务和拍 摄任务，在此期间还需要躲避障碍物和处理应急 事件 ${ }^{[1]}$. 因此，自动拍摄动态场景的无人机系统必 须拥有感知能力，用于处理跟踪目标和场景环境. 前者可以采用全球卫星定位系统 (global positioning system, GPS)和惯性测量单元(inertial measurement unit, IMU)组合导航系统 ${ }^{[12-15]}$, 视觉系统 ${ }^{[16]}$ 或动作捕捉系统 ${ }^{[15,17]}$ 进行处理; 后者一般利用一 张空间地图表示. 此外，也有一些方法利用一个虚 拟相机模仿运动物体和无人机相机之间的关系. Galvane 等 ${ }^{[15]}$ 设计了一个嵌人维持无人机与运动物 体相对关系约束的复曲面空间. Bonatti 等 ${ }^{[16]}$ 关注位 于屏幕上的演员，并设计了一个预测未知环境中 演员运动轨迹的无人机摄影框架.

\section{2 单无人机追踪单一动态目标算法}

单无人机追踪单一动态目标是使用一架无人 机对运动单一目标进行持续拍摄，同时保证无人 机与目标物体的相对位置固定, 拍摄效果良好. 单 对单追踪是对复杂动态场景进行多无人机摄影的 基础. 凌晨无人机和多无人机 2 种拍摄方式的基础 构图规则、相机布置的原则以及无人机跟踪运动时 的运动学原理是一致的. 所以，研究多无人机协同 摄影算法之前, 首先要设计一种性能优秀的单对 单追踪算法.

\section{1 针对单一目标的摄影构图设计}

不考虑光照条件，默认相机视场中心指向场 景中心，相机位置与物体位置连线与水平方向夹 角为向下 $45^{\circ}$, 完成一个俯视, 该角度可以调节. 这样进行拍摄, 可以保证场景中心占据照片的中 心位置，而俯视更符合人们使用无人机的直观感

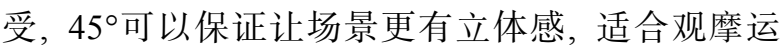
动中物体的细节 ${ }^{[18]}$.

在俯视角确定的条件下，无人机的高度直接 决定了相机的高度以及相机与拍摄物体的相对位 置，进而决定了拍摄物体在照片中呈现的大小，原 则上，在不损失关键物体信息和确保安全的基础 上，对某个场景的拍摄越近越好. 对于一个体积有 限的单一物体，自然是考虑在安全距离下越近越 好. 但是对于一个多个物体构成的小场景而言, 希 望物体都能保持在照片的中央部分. 因此, 采用
$1 / 6$ 构图法, 将照片上下左右各 $1 / 6$ 长度部分视为 边缘区域，而中间 $2 / 3 \times 2 / 3$ 的部分视为中央区域， 如图 1 所示. 在设计多物体的摄影构图时, 尽量让 所有物体都被放置于中央区域，在不损失关键物 体信息的同时，保证所有物体能尽量就近观看.

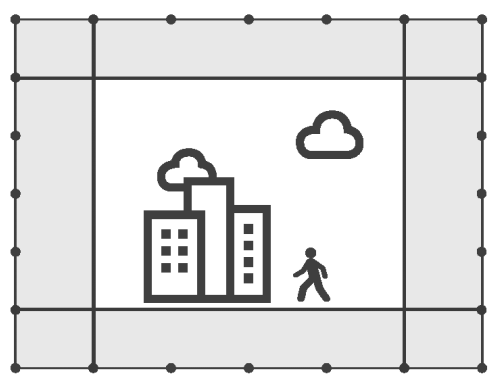

图 $11 / 6$ 构图法

采用上述设计原则, 首先根据场景范围的直 径 $d$ 、相机的视场角 FOV 、相机预设的俯视角度 $\alpha$ 确定无人机的高度 $h$; 然后根据 $h$ 和 $\alpha$ 计算出场 景中心和相机的相对位置, 再通过场景中心在场 景中的位置, 计算无人机的具体位置. 如果不考虑 物体摄影方向的需求, 根据预设的拍摄构图规则, 理想的拍摄位置点的集合为以物体中心 $O$ 在平面 $z=h$ 上的投影 $O^{\prime}$ 为圆心, 以 $h / \tan \alpha$ 为半径, 在平 面 $z=h$ 上的圆 $C$, 如图 2 所示.

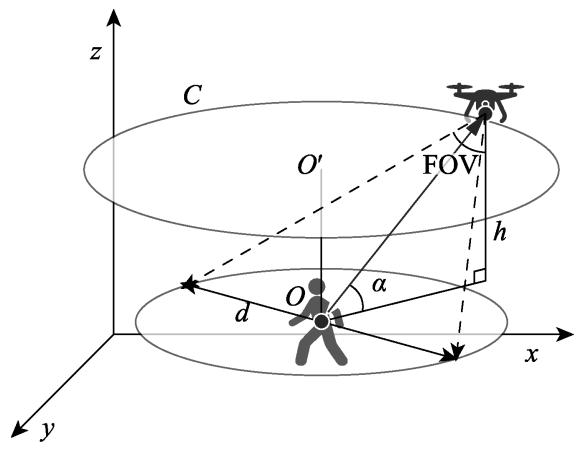

图 2 理想拍摄位置示意图

\section{2 对单一动态目标的无人机跟踪运动}

在确认拍摄构图规则后，可以根据跟踪物体 或场景的当前位置 $O$ 、无人机位置 $P$ 以及就近移 动原则, 计算出无人机飞行的目标位置 $D$. 如图 3 所示, $D$ 为 $O P$ 连线在圆 $C$ 所在平面上的投影 $O^{\prime} P^{\prime}$ 与圆 $C$ 的交点, $P D$ 为无人机飞到理想拍摄位置的 最短路径.

若能持续实时地获得当前时刻的目标位置 $D$, 就可以动态更新该目标位置, 实时指导无人机对 其跟踪飞行. 按照这样的运动方式, 每次发给无人 
机新的运动指令时, 无人机都会飞向距离无人机 最近的理想拍摄位置, 从而一直朝向物体位置运 动，形成对物体的追踪，如图 4 所示.

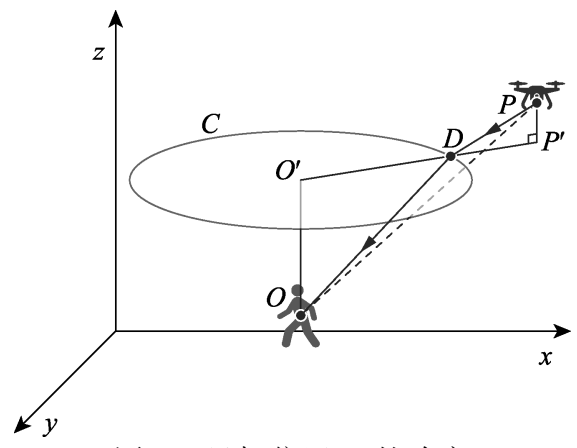

图 3 目标位置 $D$ 的确定

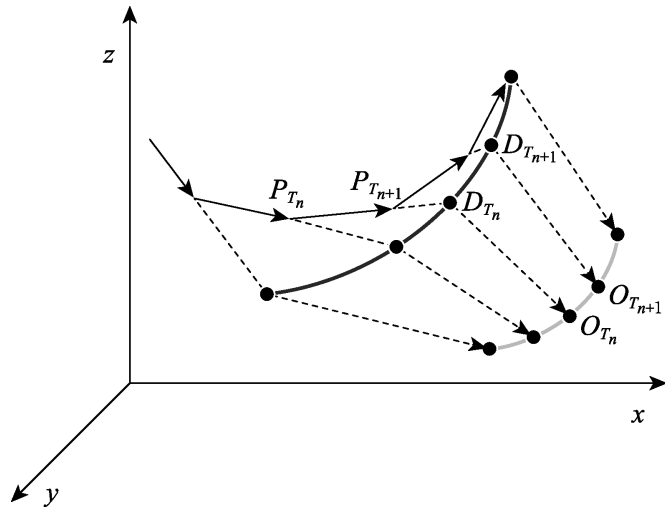

图 4 无人机跟踪示意图

\section{3 本文算法}

由于一个复杂动态场景相对无人机拍摄范围 比较大, 场景中需要拍摄的目标物体动态不定且 数量较多, 通常是无法用单架无人机独立完成全 景拍摄的, 也无法通过简单地增加无人机的数量 完成对场景中所有重点关注对象的拍摄. 在这种 条件限制下，用户仍希望能完整、实时地对场景进 行拍摄, 并且希望多架无人机拍摄的画面相互之 间有所关联，让用户既可以观察到每个重点对象 的运动细节, 又可以将不同无人机的拍摄画面相 互联系起来. 与此同时, 还要综合考虑无人机的飞 行代价, 尽量用少量的无人机进行短距离飞行, 希 望在飞行过程中, 无人机群的飞行路程总和是最 短的. 综上所述, 拍摄算法要满足以下 4 个条件.

(1) 所有关注对象都能被拍摄到，且尽量处于 相机画面的中心区域.

（2）场景能被整体地拍摄到，每个相机的画面 都能表现出它在拍摄场景的某一部分，而不是毫
无关联的松散场景画面.

(3) 尽可能地使用少量的无人机, 总飞行最短 路程完成拍摄过程.

（4）实时拍摄，尽量做到稳定、准确.

根据以上 4 个条件，本文设计的算法如下.

首先，用聚类算法将拍摄场景分割为多个小 场景，每个小场景都包含一些关注的运动对象，聚 类中心就是需要拍摄的场景中心，聚类大小就是 期望的拍摄范围大小. 其次，根据预设好的摄影构 图规则确定相机的位置, 进而确定无人机的目标 位置. 根据每个小场景的中心与整个复杂场景中 心的位置关系, 重新选定唯一的最佳拍摄位置, 以 保证在满足各个小场景的拍摄需求的同时, 也能 拍摄到整体场景的一部分, 表现出一定的关联性. 最后，根据无人机当前的位置和计算出来的无人 机目标位置, 使用指派算法进行任务指派, 尽可能 地保证无人机飞行路程总和最小, 并利用指派的 结果控制无人机飞行. 而无人机要做到实时、稳定 和准确, 则主要依靠无人机控制程序的编写, 不在 算法讨论的范畴内. 整个流程如图 5 所示.

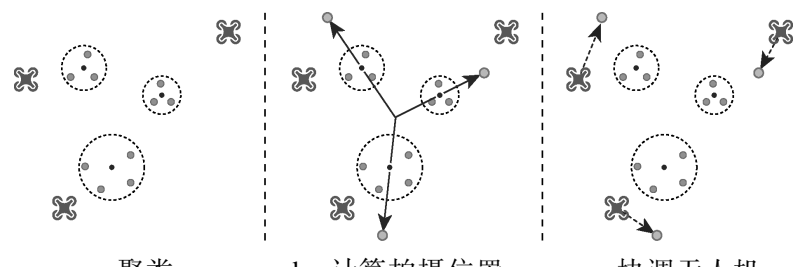

a. 聚类

b. 计算拍摄位置

c. 协调无人机

图 5 多无人机协同摄影算法流程示意图

\section{1 阈值的自适应 $\boldsymbol{K}$-means 聚类算法}

对于场景分割问题，有多个思路，其一是将场 景中所有的物体用一个凸包包围, 然后分割该凸 包. 这样做的好处是分割出的区域必然连续, 以此 为基础设计的拍摄方式，不会把场景割裂; 相对 地, 缺点也很明显, 在分割过程中, 凸包内的物体 信息相当于被隐藏了, 分割无法反映出场景内关 键物体的分布关系，极端情况下可能出现一个没 有任何关键物体的空洞. 其二是直接对场景内的 选定物体进行聚类，根据聚类结果设计无人机的 拍摄位置以及拍摄角度. 这种方式的优点是根据采 用的聚类方法, 可以保证所有的关注点都属于某一 聚类; 缺点是聚类结果之间往往没有关联性, 不控 制拍摄角度的话, 小场景的表现往往非常分散. 这 需要通过控制无人机的相机位置达成关联性.

确定采取聚类算法之后，也考虑了多种聚类 算法，包括 $K$-means ${ }^{[19]}$, Meanshift ${ }^{[20]}$, DBSCAN ${ }^{[21]}$ 


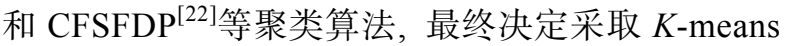
算法. 因为对于本文所处理的复杂动态场景, 物体 数量一般为几个到几十个, 数量较少而稀疏, 使用 根据密度的算法很难确定密度，且照片的拍摄区 域是一个近似于矩形的四边形，可以尽可能地与 圆形的聚类结果贴合. 如果聚类结果是凹多边形 或长条状等不规则形状，则可能会浪费很多拍摄 区域，而且难以通过划定聚类大小的方法保证聚 类结果能被包含在相机的最大拍摄范围中.

原 $K$-means 算法 ${ }^{[23]}$ 的核心思想是：计算每个 簇的样本均值作为该簇中心的新位置, 然后将每 个样本点重新分配到距离它们最近的中心所在簇, 反复迭代至聚类稳定 ${ }^{[19]}$. 可以发现，原 $K$-means 算 法具有以下 3 个缺点.

（1）无法自适应聚类，必须人工指定有几个类 或聚类中心.

(2) 聚类开始时，随机挑选聚类中心，挑选结 果不同, 最后的聚类结果也会有偏差.

(3) 只能反映聚类对象距离之间的因素，聚类 结果通常为球形, 无法通过密度等标准进行聚类, 而且没有对聚类大小进行限制，可能会产生一个 过大的聚类.

本文采取了带有國值的自适应 $K$-means 算法, 这种方法预先设定阈值，根据阈值自动计算出场 景内可以分出多少聚类，完成自适应过程; 然后对 数据集进行确定了起始点和聚类个数的 $K$-means 聚类，这样的聚类结果也是稳定的 ${ }^{[24]}$. 阈值可以 对应为期望聚类的最大范围，也就是相机的有效 拍摄范围. 样本的特征值为场景中需要关注的运 动物体的具体位置，在后续的仿真模拟飞行过程 中直接从场景程序获取该位置, 相当于在实际环 境中对关注物体上加装信号源或标记物，获取其 实际位置

该方法的原理是，选取一个初始簇中心并给 定预设阈值，剩余的簇中心根据距离和阈值一一 确定, 以获得一组初始簇中心; 然后进行 $K$-means 聚类. 每一个新簇中心的选取标准为: 若一个非簇 中心点与最近的簇中心的距离是所有非簇中心的 点中最大的, 则它即为候选的新簇中心; 然后比较 该距离与阈值的大小，小于阈值意味着当前样本 集中任意点距离其最近簇中心的距离都小于國 值，可以归属到某一个现有簇中，不必产生新的 簇中心; 大于阈值则意味着该点可作为独立的簇 中心. 这样选出来的簇中心, 保证了距离现有簇 中心最远，且簇中心之间的距离大于阈值. 具体
步骤如下.

Step1. 输人 $N$ 个样本值, 确定距离阈值 $T$. 选择样 本集 $N$ 的均值作为第 1 个簇中心 $C_{1}$, 现有簇个数 $K=1$.

Step2. 依次计算每个点与已知所有 $K$ 个簇中心的 距离, 并且选择其中的最小距离作为这个点的标记值, 记为 $d_{i}$.

Step3. 在所有点的标记值中选取值最大的点, 如 果该点满足 $d_{\text {max }}>T$, 说明该点是距离现所有聚类中心 最远的一个点, 将它作为新增的簇中心 $C_{k+1}$, 簇个数 $K=K+1$, 转 Step2; 否则, 说明所有点都在聚类中心的阈 值范围内，结束簇中心的选取.

Step4. 输出得到的 $K$ 个簇中心. 以此作为对样本 $N$ 进行 $K$-means 聚类处理的初始簇中心.

采用上述方法聚类，可以确保所有样本点都归 属某个簇，不会遗漏，且聚类的范围不会超过距离 阈值 $T$, 不会有过大聚类的问题. 过程如图 6 所示.

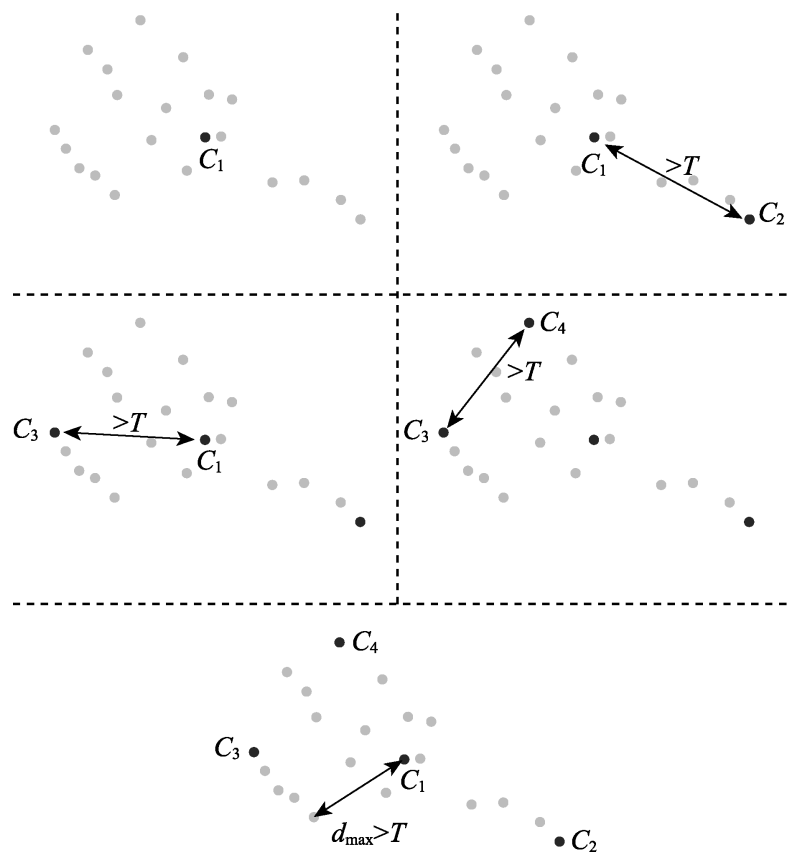

图 6 初始簇中心选取示意图

\section{2 复杂场景的摄影构图设计}

对于一个大场景，在已经通过聚类完成场景 初步分割之后, 并不能直接根据聚类的结果进行 拍摄, 否则拍摄结果会变成分散的独立小场景. 在 这个基础上, 还需要根据场景的范围和位置设定 无人机位置. 无人机的位置主要考虑高度和其在 平面上的位置 2 个方面. 高度根据设定的无人机拍 摄角度, 以及无人机与拍摄目标点的理论距离确 定，这与第 2.1 节中所述的摄影构图是类似的. 无 人机与拍摄目标的理论距离, 则根据相机的参数 计算. 根据相机的视场角, 无人机飞行时的安全高 
度以及实际拍摄中所需要拍摄场景的大小用于确 定相机高度的范围, 在此范围内, 希望无人机尽量 靠近要观察的目标位置, 同时要观察的聚类不能 超出拍摄的中心区域. 这样对于一个特定的聚类 目标，可以直接根据其聚类半径计算对其拍摄的 理论高度.

理论最适应距离和理论最适应高度计算公式 分别为

$$
\begin{gathered}
D^{\prime \prime}=\frac{R}{S \times \tan (1 / 2 \times F)} \\
H^{\prime \prime}=D^{\prime \prime} \times \sin \theta \\
H^{\prime}=\max \left(H_{\text {min }}, H^{\prime \prime}\right) \\
H=\min \left(H^{\prime}, H_{\max }\right)
\end{gathered}
$$

其中， $R$ 为聚类的半径; $S$ 为照片的中心区域所 占比例; $F$ 为相机的视场角; $\theta$ 为设定的无人机拍 摄角度； $H_{\text {min }}$ 和 $H_{\text {max }}$ 分别为无人机飞行的最低高 度和最高高度; $H$ 为求出的无人机飞行理论高度.

获得无人机高度后, 需要根据这个高度和已 知聚类中心的位置计算无人机的平面位置. 由于 希望在拍摄关注对象的同时能覆盖整个场景, 并 且对整个场景有完整立体的观感，因此无人机会 从场景的外部向场景中心进行拍摄. 具体过程如 下．首先计算出场景的中心，由于各个小场景对于 拍摄是平等的，因此取各个场景位置的算数平均 值作为场景中心即可. 然后, 将场景中心与各个场 景位置进行连线, 无人机的水平位置就在该射线 的延长线上. 根据计算所得无人机的高度和无人 机的拍摄角度, 可以计算出无人机与其拍摄场景 的水平距离为多少，在该延长线上根据水平距离 截取的位置就是无人机的位置, 如图 7 所示, 其中 $A, B, C$ 为场景位置, $A^{\prime}, B^{\prime}, C^{\prime}$ 为对应无人机位置.

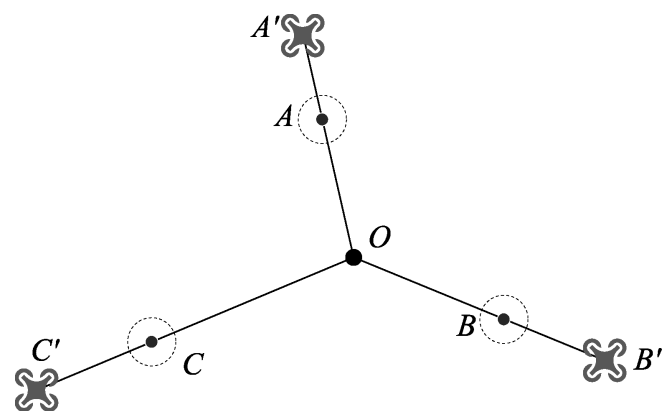

图 7 无人机平面位置示意图

场景中心位置计算公式为

$$
O=\frac{1}{K} \times \sum_{i=1}^{K} X_{i}
$$

$$
\frac{X_{i}^{\prime}-X_{i}}{X_{i}-O}=\frac{H_{i} \times \cot \theta}{\left|X_{i}-O\right|}
$$

其中, $X_{i}$ 为需要拍摄的场景位置; $K$ 为分割后的 场景中心个数; $X_{i}^{\prime}$ 为无人机理论水平位置; $H_{i}$ 为 无人机高度; $\theta$ 为拍摄俯角.

\section{3 多无人机飞行调度规划}

获得了无人机群的目标位置之后, 就可以根 据各架无人机的当前位置和目标位置分配无人机 的飞行任务. 对于多对多的无人机飞行任务分配 问题, 可以转化为一个任务指派问题. 每架无人机 到各个目标点的距离可以近似为每个任务的代价. 本文希望这个任务是完全匹配的，即调用的无人 机和目标场景位置是一对一的，这样才能完成对 场景的完整拍摄. 在实际匹配过程中，会根据聚类 结果的场景数量动态调用无人机, 如果无人机数 量不足, 则该场景无法进行完整拍摄, 只能通过增 加无人机个数或更改无人机的视野范围进而更改 聚类的距离阈值完成拍摄

对本文中的无人机指派问题的描述方法如下. 有 $m$ 个目标位置, 现在可以调用 $n$ 架无人机去拍 摄, 调用第 $i$ 架无人机飞向第 $j$ 个目标点的距离为 $d_{i j}$, 也代表了该段飞行的成本. 通常, 无人机的总 飞行距离最短, 则飞行成本最低. 根据 $m$ 和 $n$ 的不 同，指派问题可分为 3 种情况进行论述 ${ }^{[25]}$.

(1) 当 $m=n$ 时, 即为每架无人机都分配一个 目标点, 也称为完全匹配.

(2) 当 $m>n$ 时, 即目标点的数量大于无人机 的数量. 这时可虚设 $m-n$ 架无人机构成一个 $m \times m$ 的成本矩阵, 并且这 $m-n$ 架无人机飞往 $m$ 个目标的距离为飞行成本的上限.

(3) 当 $m<n$ 时, 即无人机数量大于目标点数 量时, 应虚设 $n-m$ 个目标位置, 此时目标位置没 有具体位置, 但是任意无人机飞往该虚拟目标位 置的距离成本为 0 , 即无人机可以不移动.

通过虚设目标位置或人，任意的指派问题都 能转化为完全匹配的平衡指派. 为每架无人机和 目标位置对定义一个二值函数

$$
X(i, j)=\left\{\begin{array}{l}
1, \mathrm{UAV} i \text { fly to position } j \\
0, \mathrm{UAV} i \text { fly to anothert position } k \neq j
\end{array}\right.
$$

指派问题的数学模型 ${ }^{[26]}$ 为

$$
z=\min \sum_{j=1}^{m} \sum_{i=1}^{n} X_{i j} D_{i j}
$$

其中, 


$$
\begin{gathered}
\sum_{i=1}^{n} X_{i j}=1,0<j \leqslant m \\
\sum_{j=1}^{m} X_{i j}=1,0<i \leqslant n
\end{gathered}
$$

式(8)为无人机的代价函数 $z$; 式(9)表示任一 无人机只能飞往一个目标位置; 式(10)表示任一目 标位置也只能有一架无人机飞往，二者结合即代 表无人机与目标位置的一对一约束. 这样，这个问 题就转化为一个 0-1 规划问题, 可以使用由匈牙利 算法发展而来的 $\mathrm{KM}$ 算法求解.

由飞行成本 $d_{i j}$ 构成的矩阵在指派算法中称为 效率矩阵, 平衡后该矩阵为一个 $n \times n$ 或 $m \times m$ 的矩 阵, 下文统一用 $n \times n$ 表示. $\mathrm{KM}$ 算法依据的原理为 在效率矩阵中任何行或列加上或减去同一常数并 不改变分配方案, 其基本思路是在保证效率矩阵 中元素不能为负的前提下，对效率矩阵作适当变 换, 使矩阵中包含 $n$ 个处于不同行不同列的零元 素; 然后选取这些元素形成结果矩阵 $\boldsymbol{R}$ 表示指派, 结果矩阵中对应被选中的零元素位置设为 1 , 其他 元素设为 $0^{[27]}$. 之所以选取不同行且不同列的零元 素，是因为每个指派是一对一的.

该问题的难点在于，如何通过变换生成一个 包含 $n$ 个不同行不同列的零元素, 这一步骤难以一 䟣而就. 在具体实现过程中, 首先将效率矩阵转化 为至少每行每列都有零元素的矩阵, 然后对其进 行试指派, 如果无法选出 $n$ 个不同行不同列的零元 素, 则继续通过增减变换增加矩阵中的零元素, 直 到矩阵中可以选出 $n$ 个不同行不同列的零元素. $\mathrm{KM}$ 算法的时间复杂度为 $O\left(n^{3}\right)$, 对于只有几十个
物体的场景，理论上是可以用于实时控制无人机 飞行的. 能否应用于实际环境中, 需要根据所使用 的无人机硬件条件进行具体分析和测试.

\section{4 协同摄影算法的仿真测试与分析}

本文采用仿真的方式对算法进行验证. 仿真 系统主要由 2 部分组成, 分别是在 AIRSIM-UE4 平 台上搭建的场景与基于 AIRSIM 的 api 编写的无人 机控制程序. 系统的开发环境为 Windows 10 操作 系统，无人机控制端程序使用的开发工具为 VS2017, 虚拟场景使用 UE4.18.3 进行开发. 开发 所使用的硬件环境为 DELL 图形工作站, Intel Xeon E5-2620 v4 CPU, 内存 32GB, 显卡为 NVIDIA Quadro M4000. 本仿真系统部署了无人机基础运 动规则、单无人机跟踪算法以及复杂动态场景的多 无人机协同摄影算法, 可以对无人机进行自由的 手动控制或令无人机执行指定的自动飞行任务.

\section{1 单无人机对目标追踪算法测试}

使用无人机对单目标追踪是对多目标集群追 踪的基础, 单无人机实时稳定地追踪单目标物体 拍摄，获得稳定的跟踪效果以及良好的画面，是实 现多无人机实时追踪多目标物体拍摄的前提. 单 目标追踪的主要衡量标准有以下 2 点.

(1) 无人机的拍摄效果，画面中心是否为追踪 物体.

(2) 无人机位置与追踪目标的相对位置.

其拍摄效果与跟踪的画面效果如图 8 所示. 这 些图片为单无人机跟踪目标物体测试时录制的视

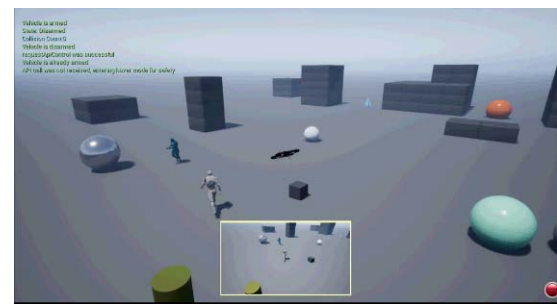

a. 开始跟踪下方画面中的白色人体模型

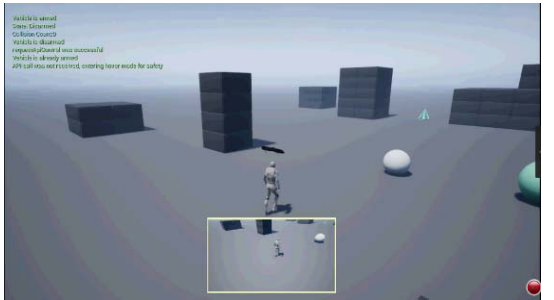

b. 跟踪 $5 \mathrm{~s}$ 后

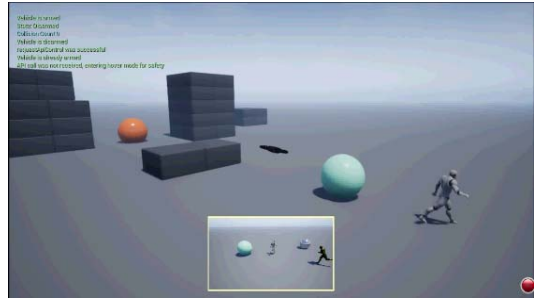

c. 跟踪 $15 \mathrm{~s}$ 后

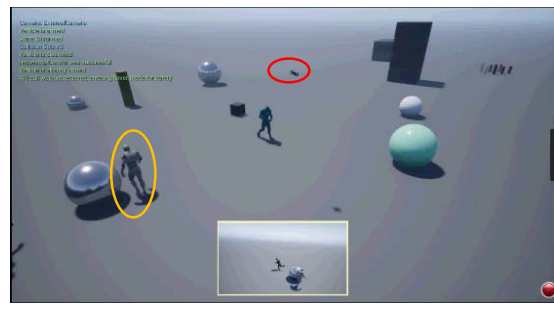

d. 从其他视角观察无人机跟踪情况

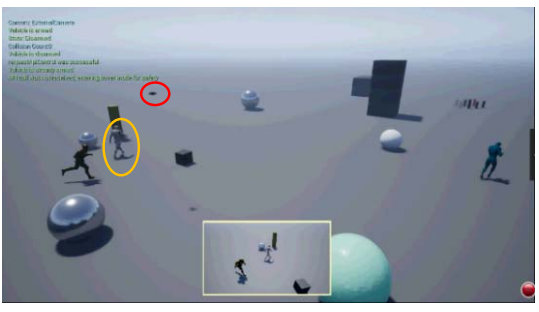

e. 在其他视角 $5 \mathrm{~s}$ 后的跟踪情况

图 8 单无人机对活动物体追踪图 
频截图，其中无人机拍摄画面为下方小框内的画 面, 跟踪物体为白色的行走人体模型, 红圈内为无 人机, 黄色圈内为跟踪的白色小人.

从图 8 中可以看到, 单无人机对目标物体的跟 踪和拍摄均有很好的结果.

开始跟踪时, 无人机位置与跟踪位置相距较 远; 之后, 无人机与跟踪目标位置快速接近, 约 $8 \mathrm{~s}$ 时会保持在 $15 \mathrm{~m}$ 左右的固定距离. 稳定跟踪 后, 当跟踪目标保持一个相对固定的方向运动时, 无人机与跟踪目标的位置差向量也会维持在一个 固定的方向和大小, 此时无人机与目标的运动接 近平行运动. 当目标出现加速、转弯、停顿等剧烈 运动变化时, 无人机与目标位置距离会出现一定 波动, 这是因为无人机运动有惯性, 且指令响应并 执行过程中有一定的迟滞性. 但是无人机仍能很 快地调整与目标的距离, 保持对目标的跟踪.

\section{2 复杂动态场景的多无人机协同摄影算法测试}

本文设计了 3 种用于测试多无人机协同摄影 的场景, 每种场景均有多个例子. 以下示例中, 中 央上部的窗口固定显示一个对整体区域的综合俯 视空中视角, 可以称之为全局视角, 用于俯政全 局, 观察其他无人机在整个场景中的位置. 其他每 个窗口显示对应的无人机拍摄画面，未被调用的 无人机对应的窗口则静止不动. 为方便观察, 示例 中所有场景的关注对象均为运动的人体模型. 测 试过程中地面上红色球体为聚类中心, 方便观察 聚类中心的移动情况. 在拍摄过程中, 会实时记录 无人机位置和聚类结果等原始数据, 记录间隔为 $1 \mathrm{~s}$, 并根据该数据绘制无人机轨迹图. 无人机轨迹 图中, 实线代表无人机的飞行轨迹, 虚线代表无人 机与其对应的聚类中心的连线, 也就是理想情况 下无人机相机指向的方向. 这样, 就可以结合拍摄 的图片结果、原始数据和无人机轨迹图 3 方面分析 算法的实现情况和拍摄效果.

\subsection{1 本文算法在不同场景的测试}

测试场景 1. 目标群分为多个组, 每组按照各 自的路线分别运动. 这种场景的特点是其聚类相 对固定，但聚类间的相对位置变化是随机的.

示例所用场景的运动轨迹如图 9a 所示. 该场 景中, 一共有 4 群人, 每个人群的运动模式都比较 平行, 保证聚类固定. 为了便于观察运动轨迹, 地 板没有添加景物和渲染地貌. 在实际拍摄过程中 会通过布置景物和渲染地貌让场景更具有真实性, 后续示例都如此. 此案例中, 拍摄共启动了 5 架无 人机, 实际拍摄中根据人群数量只调用了 4 架.

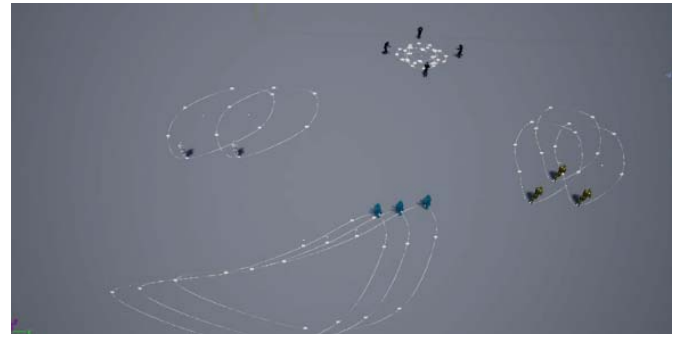

a. 固定聚类场景人物轨迹图

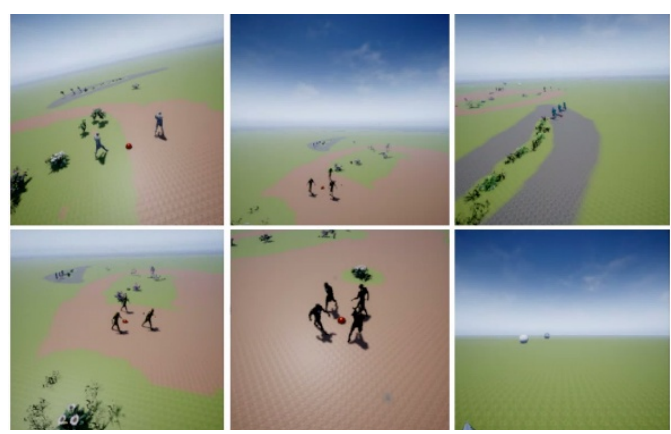

b. 飞行 $45 \mathrm{~s}$ 时的拍摄效果

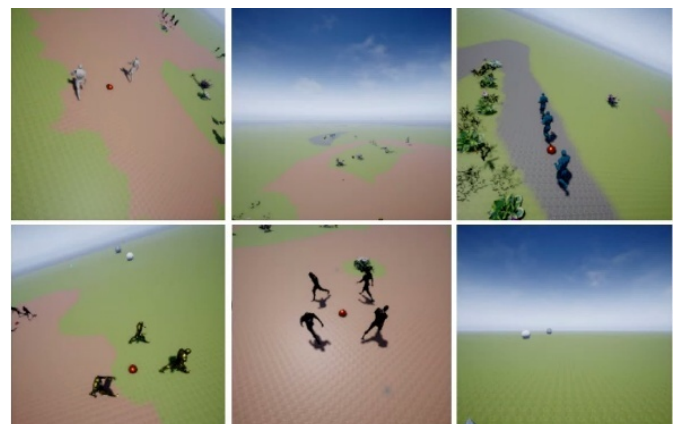

c. 飞行 $60 \mathrm{~s}$ 时的拍摄效果

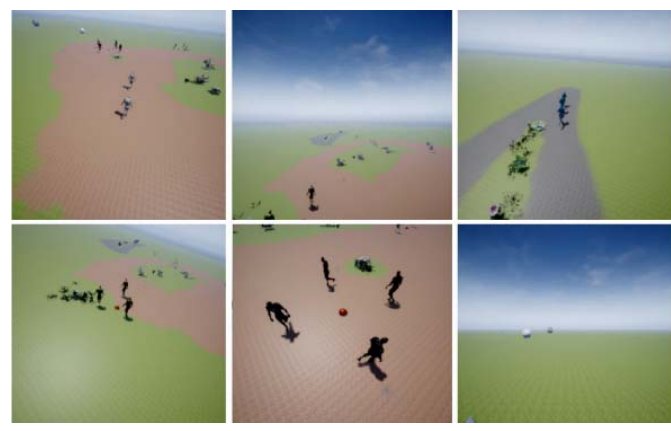

d. 飞行 $90 \mathrm{~s}$ 时的拍摄效果

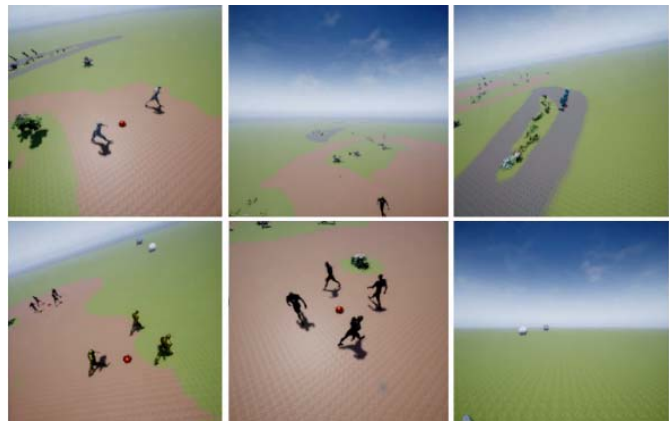

e. 飞行 $150 \mathrm{~s}$ 时的拍摄效果

图 9 固定聚类场景的多无人机协同摄影测试 
根据拍摄截图、飞行的原始数据以及如图 10 所示的轨迹图分析, 无人机在 $10 \mathrm{~s}$ 和 $20 \mathrm{~s}$ 这种较长 间隔时间后，仍能够持续对聚类中心进行重点拍 摄，无人机位置、聚类中心位置以及场景中心的位 置大体上保持了第 4 节所述的拍摄构图标准. 图 9 中，几个聚类的拍摄效果良好，没有终点目标遗 漏，同时几架无人机拍摄的画面也都指向场景中 心，不再是独立的画面，很有整体感. 由轨迹图可 知, 无人机群从一个相距较近的起点出发, 当无人 机开始飞行 $25 \mathrm{~s}$ 左右后, 会飞到场景附近的位置. 由于该场景运动比较稳定, 因此物体与聚类中心 的距离和方位也会相对固定，拍摄效果也会趋于 稳定. 经测试，同类型的其他场景也都与上述结果 类似. 由此, 对于固定聚类的场景，该算法可以获 得很好的拍摄效果.

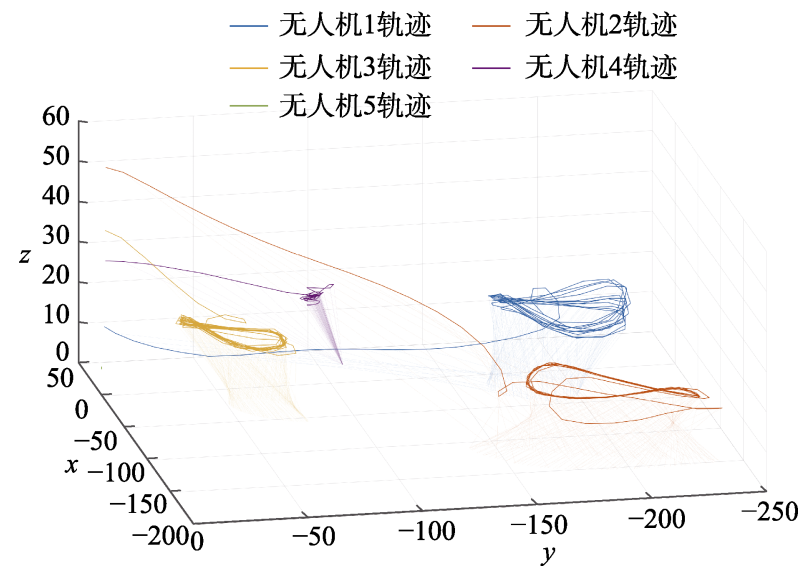

图 10 固定聚类多无人机协同摄影飞行轨迹图

测试场景 2. 多个目标朝向或背离一个中心点 运动, 能在一个循环周期内聚到中心, 然后会各自 走向不同位置，往复运动，其聚类表现为时聚时 散. 当所有目标聚到一起时, 聚类结果只有一个; 当散开时, 会产生多个聚类中心.

本示例所用场景的运动轨迹如图 11a 所示. 该 场景为 4 个人物目标进行循环往复运动, 开始时都 从中心向外运动，其中每 2 个人朝向一个方向，另 外 2 人朝向另一方向, 行走一段距离后, 每个小组 又自行分开，几个人的距离超过了无人机设置的 拍摄范围阈值，每个目标都变成一个单独的聚类。 随后，4 个人又会朝向中心，一起向内运动，变回 一个聚类，构成一个循环. 聚类中心的个数变化为 $1 \rightarrow 2 \rightarrow 4 \rightarrow 2 \rightarrow 1$. 本案例中, 总共启动了 4 架无人 机，在拍摄过程中会动态调用.

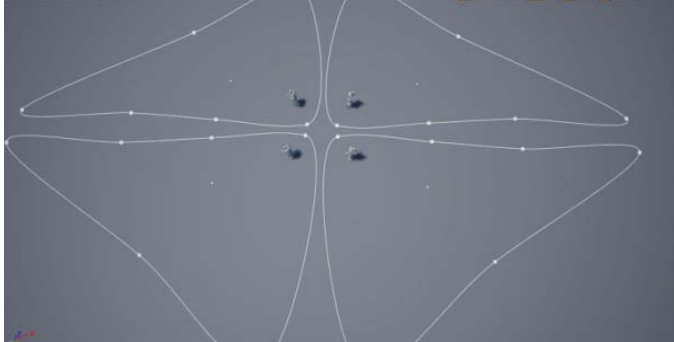

a. 时聚时散场景人物轨迹图

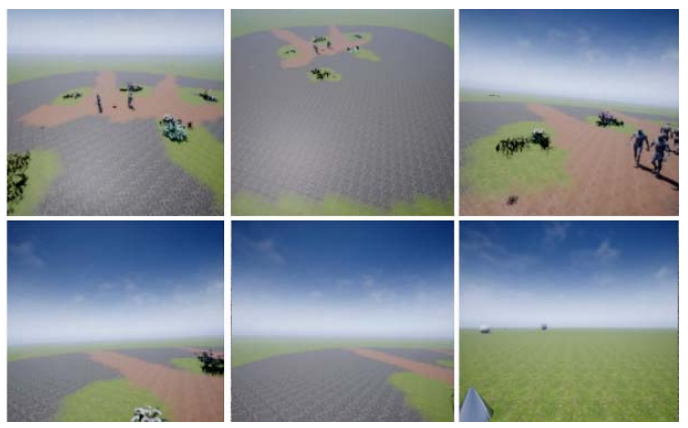

b. 飞行 $60 \mathrm{~s}$ 时的拍摄效果

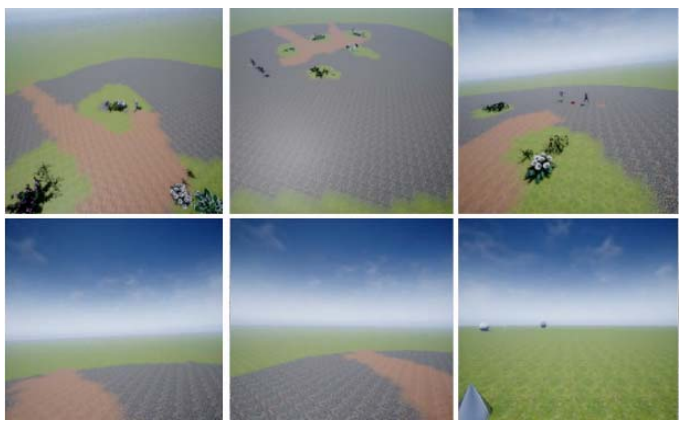

c. 飞行 $75 \mathrm{~s}$ 时的拍摄效果

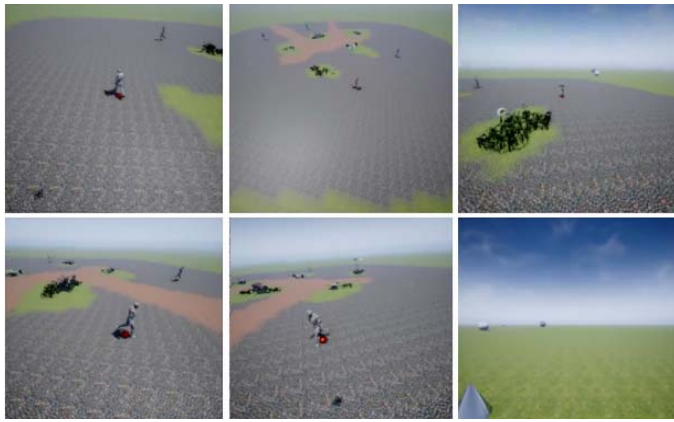

d. 飞行 $90 \mathrm{~s}$ 时的拍摄效果

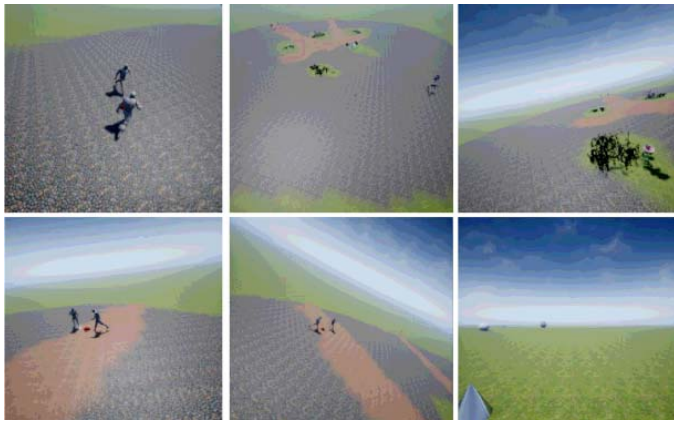

e. 飞行 $105 \mathrm{~s}$ 时的拍摄效果

图 11 时聚时散场景的多无人机协同摄影测试 
根据拍摄截图、飞行的原始数据以及如图 12 所示的轨迹图分析, 对于这种聚类中心数量会剧 烈变化的场景，本文算法可以动态调用无人机，没 有被调用的无人机在原地悬停待命，它对这种场 景是动态适应的. 图 11 中, 大部分时间拍摄效果 良好. 截图所示过程完整地反映了聚合过程, 第 1 张图为 4 个人聚集在中间, 只调用了一架无人机进 行拍摄; 第 2 张图中 4 个人走向了场景 4 个角落, 此时调用了 4 架无人机对 4 个人分别进行拍摄; 第 3 张图中，每 2 个人聚集在一起，形成了 2 组，调用 了 2 架无人机进行拍摄; 在最后 1 张图中, 4 个人又 重新聚集到了一起, 形成了一个聚合的循环. 经测 试，同类型的其他场景除了个别场景过小，导致整 个场景小于无人机拍摄范围的阈值，其他场景仍 符合上述结果. 由此，对于时聚时散类的场景，该 算法可以获得很好的拍摄效果.

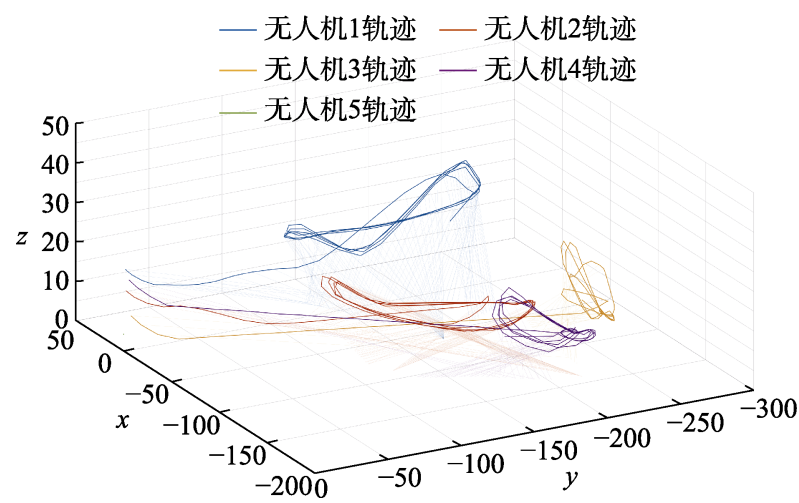

图 12 时聚时散场景多无人机协同摄影飞行轨迹图

测试场景 3. 多个目标随机运动，没有规律， 这种场景的聚类个数难以预测, 运动也不规则, 也 不会出现循环往复式的运动. 该场景拍摄难度比 较大，需要较长的拍摄时间.

本示例所用场景的运动轨迹如图 13a 所示. 其 中, 左侧有 4 个人为小聚类, 右侧有 6 个人不规则 聚散. 计算的聚类结果一般为 4 或除上述费用外的 其他费用 5 个.

本案例共启用了 5 架无人机．根据拍摄截图、 飞行的原始数据以及如图 14 所示的轨迹图分析, 对于随机运动场景，本算法仍可以动态调用无人 机，该算法对随机场景依然是动态适应的. 图 13 中，大部分时间拍摄效果良好，少部分时间会由于 聚类的剧烈变化导致无人机在短时间内重新确定 拍摄目标, 并进行紧急的加速或减速, 进而出现比

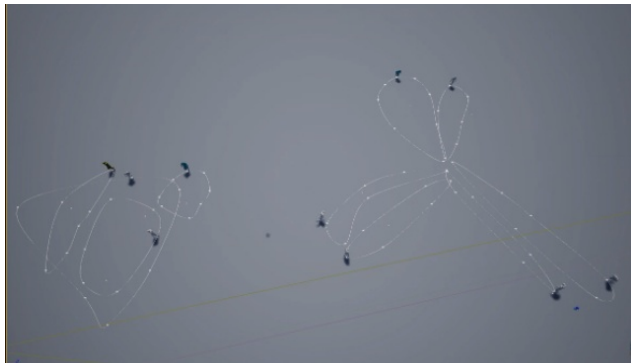

a. 随机运动场景人物轨迹图

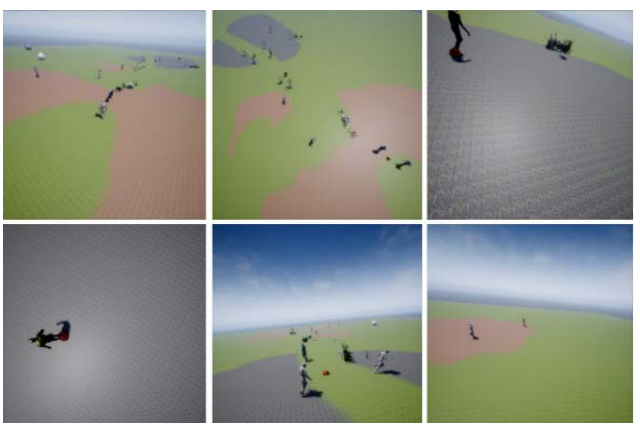

b. 飞行 $30 \mathrm{~s}$ 时的拍摄效果

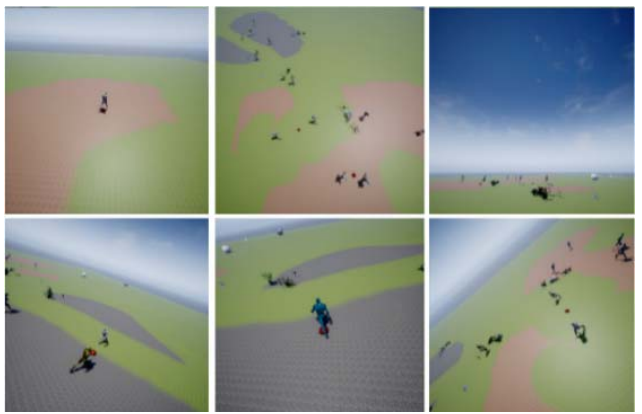

c. 飞行 $40 \mathrm{~s}$ 时的拍摄效果

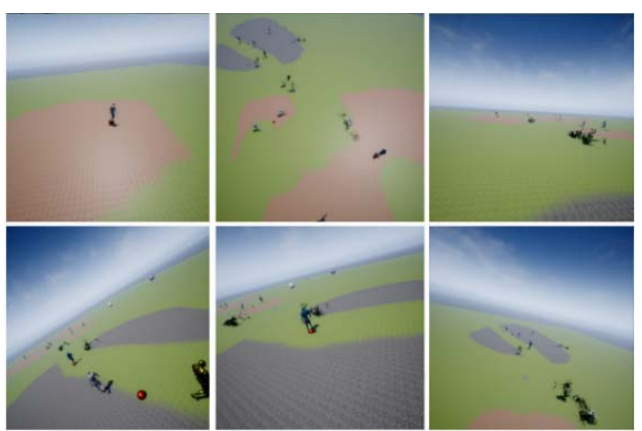

d. 飞行 $50 \mathrm{~s}$ 时的拍摄效果

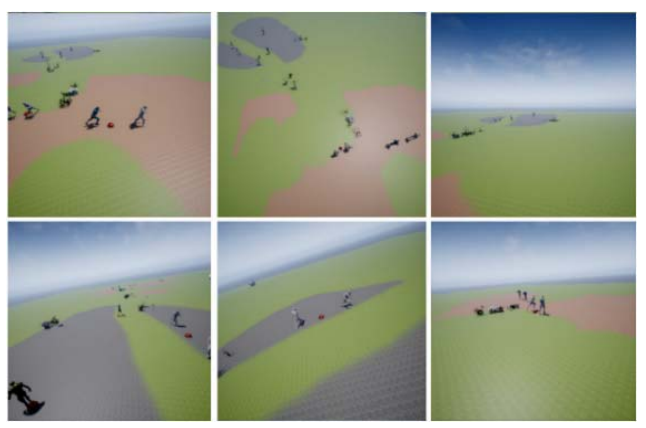

e. 飞行 $60 \mathrm{~s}$ 时的拍摄效果

图 13 随机运动场景的多无人机协同摄影测试 


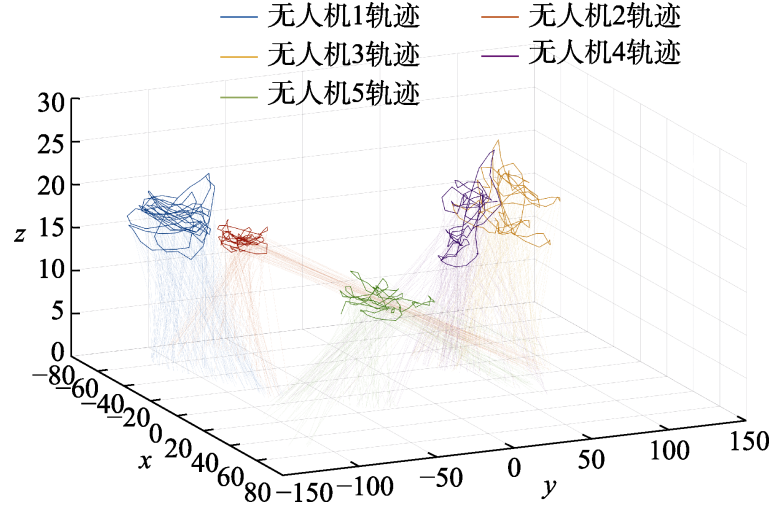

图 14 随机场景多无人机协同摄影飞行轨迹图

较严重画面抖动. 不过随着聚类中心的重新稳定, 拍摄也会重新趋于稳定. 同类型的场景情况都比 较复杂, 难以统一概括, 有一些场景可以保持平稳 有序拍摄. 由此，对于随机运动场景，该算法可以 在大部分情况下达到拍摄要求.

\subsection{2 协同摄影算法与均分法的对比}

对于一个较大的场景，如大型体育场、建筑群 等，如果想同时拍摄该场景所有部分，均分场景进 行拍摄是一种非常简单有效的方法. 但是对于复 杂的动态场景，均分法是有缺陷的. 这是因为均分 法没有考虑到场景内部物体相对的位置关系.

均分拍摄法的主要均分方式有 2 种，一种是基 于拍摄区域进行面均分; 另一种是基于拍摄方向 进行角均分. 本文将协同算法与这 2 种方法一同进 行了除上述费用外的其他费用测试. 面均分的拍 摄方式为自场景上方垂直向下拍摄，每架无人机 的拍摄区域为矩形，让多架无人机的拍摄区域覆 盖整个场景即可. 角均分为以场景中心为圆心，取 同一高度、半径，根据无人机数量均分拍摄角度， $360^{\circ}$ 环绕拍摄. 测试所采用的场景为固定聚类，示 例场景轨迹图如图 9(a), 对比效果如图 15 所示.

如图 15 所示, 面均分法调用了 4 架无人机从 上至下拍摄，角均分法调用了 5 架无人机从四周向 场景中心拍摄. 可以发现，均分法有以下缺陷.

（1）画面没有主次之分，空旷和密集区域都使 用同样的方式拍摄.

（2）运动物体可能位于摄像机拍摄的边缘，导 致物体正好被画面分割.

（3）不会根据物体的运动情况，尤其是场景内 部的物体, 调整无人机的拍摄位置与拍摄朝向，没 有特写，缺失拍摄的细节.

相比而言，协同摄影算法有以下几个优点.

（1）会对集群目标进行针对性的集中拍摄，并

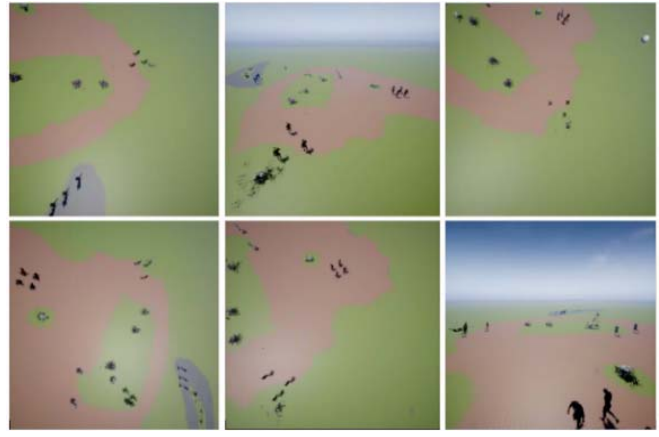

a. 面均分法拍摄效果

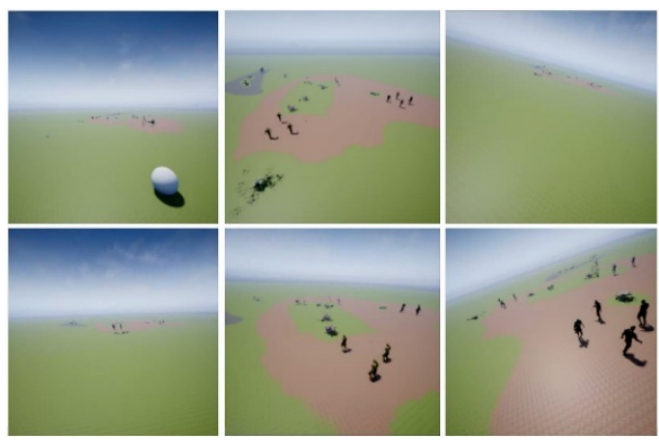

b. 角均分法拍摄效果

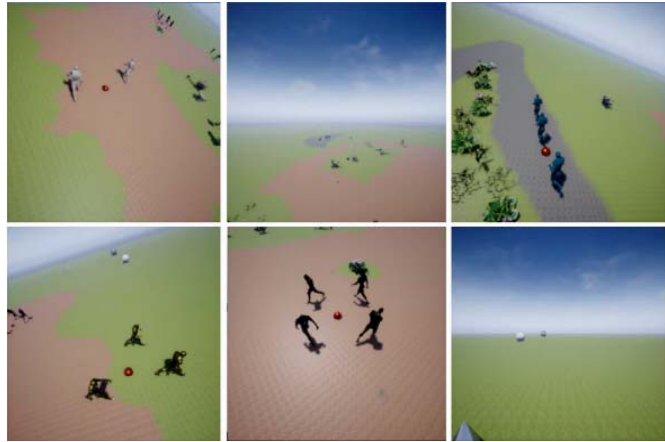

c. 多无人机协同摄影拍摄效果

图 15 多无人机协同摄影算法与均分法 拍摄效果对比

且尽量保持合理的拍摄位置, 能清楚地观看物体 运动的细节.

（2）稀疏或空白处不是拍摄的重点，会减少无 人机的分配.

（3）尽量避免重要物体被拍摄画面分割.

(4) 所有无人机会根据物体聚类移动.

综上所述，协同摄影算法比均分摄影法更适 合拍摄复杂动态场景.

\section{5 结 语}

本文从对大型复杂场景的拍摄需求出发, 介 绍了对大型场景拍摄复杂的相关算法和研究工作, 进而提出了解决方案. 采取 $K$-means 算法对场景中 
的运动对象进行无遗漏的聚类，然后根据聚类结 果计算无人机的理想拍摄位置, 最后采用匈牙利 算法实现无人机的最低飞行成本指派．并基于 AIRSIM 插件和 UE4 环境, 开发了搭载上述算法的 无人机控制程序，进行了仿真验证，仿真结果能大 体反映出算法的可用性，可以对比较复杂的动态 场景进行分割拍摄.

本文方法尚有以下不足.

（1）本文仅在 UE4 平台下进行了仿真，没有在 实际环境中进行无人机的飞行测试.

（2）在仿真飞行测试中，实际上物体的位置不 是通过计算机视觉计算得来的，而是直接通过取 位置实现的，相当于实际环境中在物体上加装了 一些标记物或信号源, 能直接获取其物理位置.

(3) 飞行过程中, 无人机的飞行位置实际上是 有一定误差的，该误差难以通过无人机运动过程 中的控制完全消除.

（4）由于需要进行实时性的拍摄，以及无人机 飞行有一定误差, 无人机在加减速过程中往往会 有剧烈的抖动, 影响拍摄效果. 对随机运动的场景 进行拍摄时，这种情况比较明显.

(5) 当聚类中心数量有陡变时, 会导致调用的 无人机与聚类数目不匹配. 本文在设计场景时，尽 量保证可供调用的无人机多于聚类数目, 避免遗 漏. 而在拍摄过程中, 若有多余的无人机，目前采 取的策略是原地待命, 这样的处理方式比较简单 直接, 便于再次需要使用无人机时进行调用, 但是 用户观感比较差, 难以区分有命令和无命令的无 人机. 对聚合场景拍摄时, 这种情况比较明显.

针对以上不足，提出以下改进方向.

(1) 根据真实的拍摄需求，可以使用多架无人 机进行实际飞行测试. 但须考虑多架无人机的不 同相机参数, 这与现有 AIRSIM 的环境设置不同.

(2) 可以根据无人机拍摄的画面, 使用机器视 觉的方法计算目标物体的位置.

(3) 对 AIRSIM 源程序进行更深人的分析，修 改 AIRSIM 的底层代码，以对仿真环境中的无人机 进行更精确的控制.

（4）改进现有的无人机调度规划算法，以解决 无人机与聚类之间数量不匹配的问题. 大体思路 如下: 若有多余的无人机，将它们安排到场景旁边 的无关位置并控制相机的拍摄内容; 当无人机数 量不足时，对各个场景进行评估，挑选重要的场景 进行拍摄.

\section{参考文献(References):}

[1] Capitan J, Torres-Gonzalez A, Ollero A. Autonomous cinematography with teams of drones[OL]. [2020-08-29]. https:// personales.us.es/jcapitan/preprint/capitan_iros19_web.pdf

[2] Mademlis I, Mygdalis V, Nikolaidis N, et al. Challenges in autonomous UAV cinematography: an overview[C] //Proceedings of the IEEE International Conference on Multimedia and Expo. Los Alamitos: IEEE Computer Society Press, 2018: 1-6

[3] Zhao Yuliang. Principles of aerial photography[M]. Beijing: National Defense Industry Press, 2017: 5-14(in Chinese) (赵育良. 航空摄影原理[M]. 北京：国防工业出版社，2017: 5-14)

[4] Bi Kai, Li Yingcheng, Ding Xiaobo, et al. Aerial photogrammetric technology of light small UAV: status and trend of development[J]. Bulletin of Surveying and Mapping, 2015(3): 27-31+48(in Chinese)

(毕凯, 李英成, 丁晓波, 等. 轻小型无人机航摄技术现状及 发展趋势[J]. 测绘通报, 2015(3): 27-31+48)

[5] Salomon B, Garber M, Lin M C, et al. Interactive navigation in complex environments using path planning[C] //Proceedings of the Symposium on Interactive 3D Graphics. New York: ACM Press, 2003: 41-50

[6] Nieuwenhuisen D, Overmars M H. Motion planning for camera movements[C] //Proceedings of the IEEE International Conference on Robotics and Automation. Los Alamitos: IEEE Computer Society Press, 2004: 3870-3876

[7] Joubert N, Roberts M, Truong A, et al. An interactive tool for designing quadrotor camera shots[J]. ACM Transactions on Graphics, 2015, 34(6): Article No.238

[8] Gebhardt C, Hepp B, Nägeli T, et al. Airways: optimization-based planning of quadrotor trajectories according to high-level user goals[C] //Proceedings of the CHI Conference on Human Factors in Computing Systems. New York: ACM Press, 2016: 2508-2519

[9] Roberts M, Hanrahan P. Generating dynamically feasible trajectories for quadrotor cameras[J]. ACM Transactions on Graphics, 2016, 35(4): Article No.61

[10] Fleureau J, Galvane Q, Tariolle F L, et al. Generic drone control platform for autonomous capture of cinema scenes[C] //Proceedings of the 2nd Workshop on Micro Aerial Vehicle Networks, Systems, and Applications for Civilian Use. New York: ACM Press, 2016: 35-40

[11] Xie K, Yang H, Huang S Q, et al. Creating and chaining camera moves for qadrotor videography[J]. ACM Transactions on Graphics, 2018, 37(4): Article No.88

[12] Joubert N, Jane L E, Goldman D B, et al. Towards a drone cinematographer: Guiding quadrotor cameras using visual composition principles[OL]. [2020-08-29]. https://arxiv.org/ pdf/1610.01691.pdf

[13] Nägeli T, Meier L, Domahidi A, et al. Real-time planning for automated multi-view drone cinematography[J]. ACM Transactions on Graphics, 2017, 36(4): Article No.132

[14] Galvane Q, Lino C, Christie M, et al. Directing cinematographic drones[J]. ACM Transactions on Graphics, 2018, 37(3): Article No.34 
[15] Galvane Q, Fleureau J, Tariolle F L, et al. Automated cinematography with unmanned aerial vehicles[OL]. [2020-08-29]. https://arxiv.org/pdf/1712.04353.pdf

[16] Bonatti R, Ho C, Wang W S, et al. Towards a robust aerial cinematography platform: Localizing and tracking moving targets in unstructured environments[OL]. [2020-08-29]. https:// arxiv.org/pdf/1904.02319.pdf

[17] Mellinger D, Kumar V. Minimum snap trajectory generation and control for quadrotors[C] //Proceedings of the IEEE International Conference on Robotics and Automation. Los Alamitos: IEEE Computer Society Press, 2011: 2520-2525

[18] An Ke, Zhang Shunxi. The tilt photographic measuration technique and expectation[J]. Survey World, 2019(4): 102-103+ 112(in Chinese)

(安可, 张顺喜. 倾斜摄影测量技术应用及展望 $[\mathrm{J}]$. 经纬天 地, 2019(4): 102-103+112)

[19] Jain A K. Data clustering: 50 years beyond $K$-means[J]. Pattern Recognition Letters, 2010, 31(8): 651-666

[20] Cheng Y Z. Mean shift, mode seeking, and clustering[J]. IEEE Transactions on Pattern Analysis and Machine Intelligence, 1995, 17(8): 790-799

[21] Schubert E, Sander J, Ester M, et al. DBSCAN revisited, revisited: why and how you should (still) use DBSCAN[J]. ACM Transactions on Database Systems, 2017, 42(3): Article No.19

[22] Rodriguez A, Laio A. Clustering by fast search and find of den- sity peaks[J]. Science, 2014, 344(6191): 1492-1496

[23] Liu Yiming, Zhang Huaxiang. Approach to selecting initial centers for $K$-means with variable threshold[J]. Computer Engineering and Applications, 2011, 47(32): 60-62(in Chinese) (刘一鸣, 张化祥. 可变阈值的 $K$-Means 初始中心选择方法 [J]. 计算机工程与应用, 2011, 47(32): 60-62)

[24] An Jiyong, Yan Ziji, Zhai Jingxuan. $K$-means clustering algorithm based on distance threshold and weighted sample[J]. Microelectronics \& Computer, 2015(8): 135-138(in Chinese) (安计勇, 闩子骥, 翟靖轩. 基于距离阈值及样本加权的 $K$-means 聚类算法 $[\mathrm{J}]$. 微电子学与计算机, 2015(8): 135-138)

[25] $\mathrm{Hu}$ Jingshuang. The model and solution of a kind of allocation problem[J]. Journal of Qingdao Technological University, 2006, 27(4): 125-128(in Chinese)

(胡京爽. 一类指派问题的数学模型及解法 $[\mathrm{J}]$ 青岛理工大 学学报, 2006, 27(4): 125-128)

[26] Wang Qionghua, Wang Gang. A solution to the modeling of assignment problem: the Hungarian way[J]. Journal of Kunming Metallurgy College, 2006, 22(5): 82-84(in Chinese) (王琼华, 王刚. 指派问题数学建模的匈牙利解法 $[\mathrm{J}]$. 昆明 冶金高等专科学校学报, 2006, 22(5): 82-84)

[27] Wang Jian. Coordination trajcctory planning algorithm for multiple unmanned air vehicle attacking multi-targets[D]. Xi'an: Northwestern Polytechnical University, 2004(in Chinese) (王健. 多架无人机攻击多目标的协同航迹规划算法研究 [D]. 西安：西北工业大学, 2004) 